\title{
Precision Robotic-Assisted Implantation for Preclinical Stereotactic Neurosurgery
}

Zheng Liu', Mojtaba Taherisadr'², Hemmings CH. Wu³, Shiva Abbaszadeh ${ }^{1}$

${ }^{1}$ Dept. of Nuclear, Plasma, and Radiological Engineering, University of Illinois at Urbana-Champaign, Urbana, IL, USA

${ }^{2}$ Dept. of Computer and Information Science, University of Michigan, Dearborn, MI, USA

${ }^{3}$ Dept. of Neurosurgery, Stanford University, 213 Quarry Road, Palo Alto, CA USA 94304-5979

\begin{abstract}
During stereotactic neurosurgery procedures, needle insertion or implantation of electrode/cannula/optic fibre requires quick, precise, and accurate identification of insertion location and proper guidance of the needle to accurately reach the target. In this study, we investigate an automated method to locate the entry point of the region of interest. This method leverages a digital image capture system, pattern recognition, and motorized stages. Region-based convolutional neural network and template matching of known anatomical identifiable regions is used to find regions of interest (e.g., Bregma) in rodents.
\end{abstract}

Keywords: Stereotactic neurosurgery; Automation; Template matching; Preclinical; Convolutional neural network; Image segmentation

\section{Introduction}

Stereotactic neurosurgical procedure is a common practice in both preclinical and clinical settings. In animal studies, researchers use it to intervene specific regions of the brain to carry out various experiments of different aims. One example is the use of optogenetics to investigate functions of different brain regions, which involves stereotactic injection of virus and stereotactic implantation of optic fibre. It is also widely used clinically as a diagnostic tool for brain tumor biopsy, and for treatment of various neurologic disorders, including pain, movement disorders (e.g., Parkinson's disease), epilepsy and psychiatric disorders.

The current state-of-the-art stereotactic procedure in rodents involves many steps of calculation and human estimation, and is not error-free. For example, one of the key step is the identification of the Bregma, an anatomical point on the skull at which the coronal suture is intersected perpendicularly by the sagittal suture, which is used as the main reference point. In theory, it is a point which researchers can pinpoint with high accuracy, but in practice, that is seldom the case (Figures 1a-1c). The sagittal suture, another important landmark, is also never a straight line.

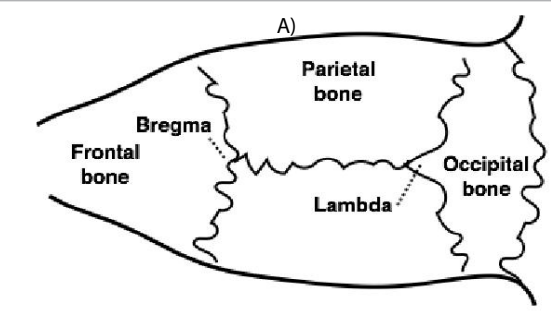

B)

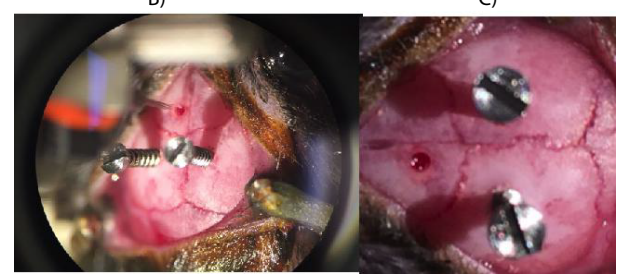

Figure 1: (a) Schematic of region of interest. (b) Angled view with the Bregma and injection site visible. (c) An example of an image used for template matching.

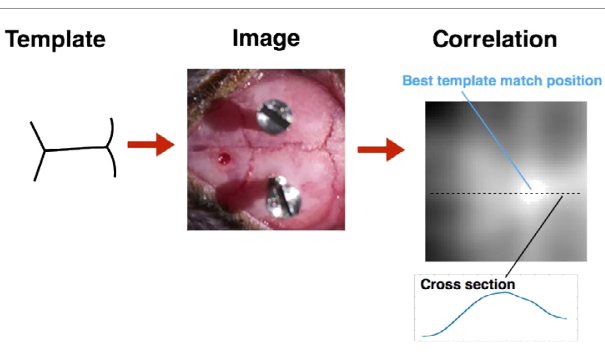

Figure 2: Example template use on an image and the associated correlation plot for a template translated over a $50 \times 50$ pixel region.

Identification of the Bregma and the sagittal suture, for example, is a part that requires repetitive checking, is laborious and timeconsuming, and can benefit substantially from automation. Previous work has investigated injection site accuracy, system automation, and registration [1-4].

In this research, we are investigating a technique to speed up this process in animal studies automatically. We present results of automatically identifying features and injection site for a stereotactic procedure in rodents. We also present the automation strategy for a potential system $[5,6]$.

\section{Methods}

Figure 2 illustrates the concept of the method used in this work. Rodents were prepared using the standard techniques for the stereotactic procedure (Figure 3). Photos of numerous rodents were acquired to get a sizeable data set. Images are firstly cropped by a region-based convolutional neural network ( $\mathrm{R}-\mathrm{CNN})$ [7], and then a

*Corresponding author: Abbaszadeh S, Department of Nuclear, Plasma and Radiological Engineering, University of Illinois at Urbana-Champaign, Urbana, IL, USA, Tel: 217-244-9738; E-mail: Sabbasza@illinois.edu

Received June 26, 2017; Accepted August 03, 2017; Published August 07, 2017

Citation: Liua Z, Taherisadr M, Wu HCH, Abbaszadeh S (2017) Precision RoboticAssisted Implantation for Preclinical Stereotactic Neurosurgery. J Appl Mech Eng 6: 279. doi: 10.4172/2168-9873.1000279

Copyright: (c) 2017 Liua Z, et al. This is an open-access article distributed under the terms of the Creative Commons Attribution License, which permits unrestricted use, distribution, and reproduction in any medium, provided the original author and source are credited. 
Citation: Liua Z, Taherisadr M, Wu HCH, Abbaszadeh S (2017) Precision Robotic-Assisted Implantation for Preclinical Stereotactic Neurosurgery. J Appl Mech Eng 6: 279. doi: 10.4172/2168-9873.1000279

template matching technique [8] is used to determine the location of the identified structure of interest. The normalized cross-correlation is used to identify the location of the optimal template match. The peak in the correlation plot corresponds to the best match with the template.

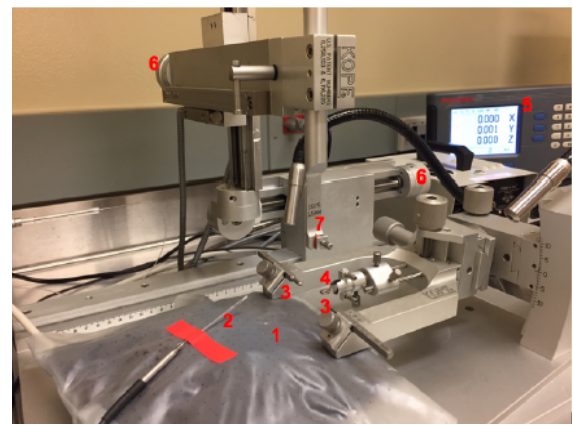

Figure 3: Preclinical stereotactic fixture: (1) heating pad, (2) body temperature probe, (3) ear pins, (4) mouth piece, (5) stereotactic coordinate monitor, (6) $\mathrm{x}$ $\mathrm{y}-$, and zknobs, (7) stereotactic arm for injection, implantation, etc.

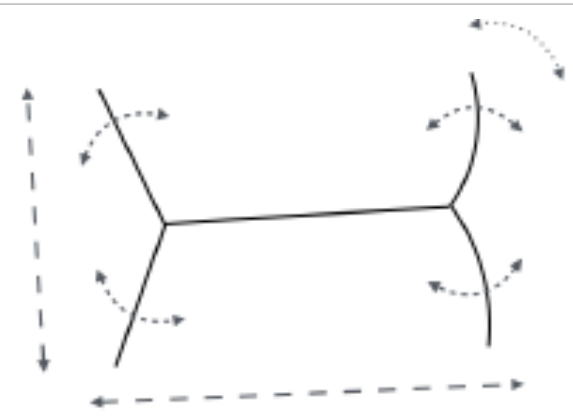

Figure 4: Variations to the template for better matching include length, rotation and thickness of the different pieces.

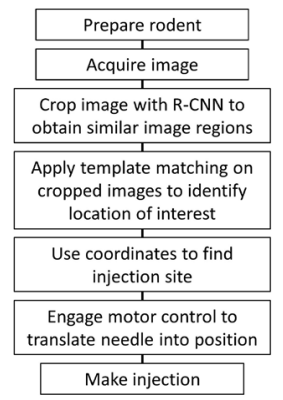

Figure 5: Flow diagram of potential automated system.

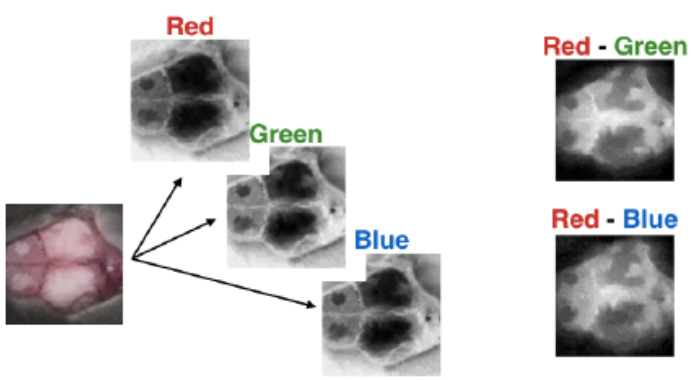

Figure 6: Example image separated into RGB components. Subtraction of Red and Green, and Red and Blue components.
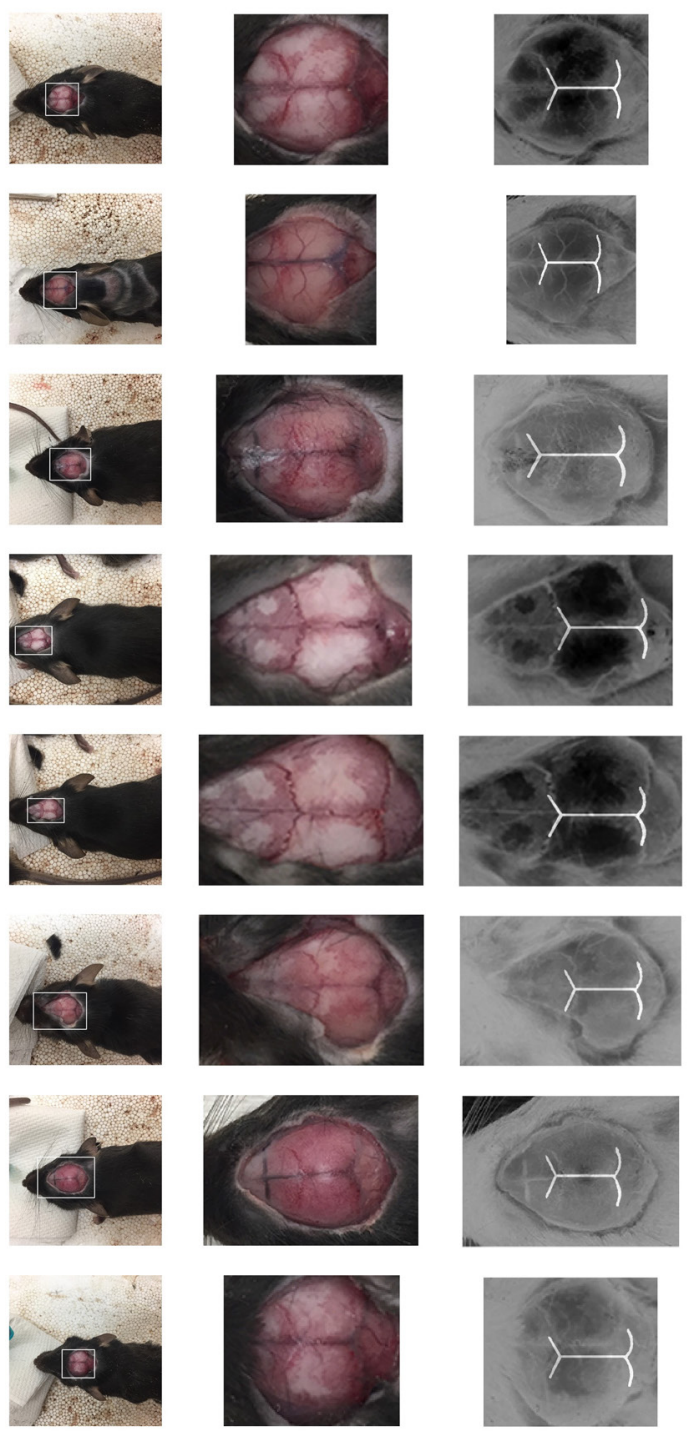

Figure 7: (left) Test images with region of interest determined by R-CNN overlaid on top of each images. (middle) Zoomed-in images of region of interest. (right) Test images with template overlaid on top at position determined using correlation plots.

This location is then used to locate the features of interest, such as the Bregma, and determine the injection site.

Template matching works best if the shape is at the same orientation and angle (example variations to the template are shown in Figure 4). Therefore, the R-CNN algorithm was used to identify the region of interest and crop the images. A total of 20 images were used to train the R-CNN model. Tensorflow was used to build the R-CNN model. In the template matching step, three templates with different length were used. For each image, the best matching template was identified and used to locate the features of interest. MATLAB (MathWorks, Natick, MA) was used for template matching.

The flow diagram of a potential automated system is shown in Figure 5, which includes both location identification as well as injection. First the rodent is prepared according to standard procedures. An image is acquired of the rodent skull. This image is cropped by the $\mathrm{R}-\mathrm{CNN}$ algorithm, and then template matching is applied to identify 
Citation: Liua Z, Taherisadr M, Wu HCH, Abbaszadeh S (2017) Precision Robotic-Assisted Implantation for Preclinical Stereotactic Neurosurgery. J Appl Mech Eng 6: 279. doi: 10.4172/2168-9873.1000279

the location of interest in the image. A virtual grid in the image is used to determine distances. A calibration step is initially carried out before the procedure to translate pixels to real space in the imaging system. The coordinates of the injection site are then fed to the motor control to move the needle into position. Lastly the injection is made.

\section{Results and Discussion}

Eight test images were first cropped by the R-CNN algorithm, and only the region of interest was retained. For these cropped images, there are 3 potential components to apply the template matching to, either the red, green, or blue channels (figure 6). For the results presented here, the template matching was applied to the blue channel. The template was translated by 100 pixels in $\mathrm{x}$ and $\mathrm{y}$ directions.

The results for eight testing images are shown in figure 7. The R-CNN algorithm cropped the region of rodent's skull in all testing images correctly. The template is then overlaid with the cropped image based on the position in the correlation plot with the highest value. In figure 7, the template matching algorithm correctly located the template on the first five images. For the last three images, the template's shape does not compatible with the images, and the locations of the templates are not accurate. This issue could be solved by trying out more templates with different shapes.

\section{Conclusion}

In this paper, we demonstrate a method for identifying anatomical features for the stereotactic procedure. This method has the potential to reduce effort and increase accuracy for a time laborious, and errorprone process. To accomplish this task a region-based convolutional neural network and a template matching method is used. The R-CNN algorithm is used to crop the images and obtain image samples with similar regions of interest. The correlation of the template with the image is used to pinpoint the location of anatomical features. A fully automated procedure is proposed to automate the location detection and injection. Future work involves optimizing the template and minimizing the separation distance between the location found using this method and the location determined by human professionals. CNN-based methods that can directly output the coordinates of interested anatomical points will also be explored.

\section{References}

1. Grange P, Pologruto T, Pinskiy V, Wang H, Khabbaz A (2010) Computer-guided stereotactic injections for the mouse brain. architecture project. Meeting of the Society for Neuroscience, Toronto, Canada.

2. Rangarajan JR, Velde GV, Van Gent F, De Vloo P, Dresselaers T, et al. (2016) Image-based in vivo assessment of targeting accuracy of stereotactic brain surgery in experimental rodent models. Sci Rep 6: 38058.

3. Henderson M, Pinskiy V, Tolpygo A, Savoia S, Grange P, et al. (2014) Automated placement of stereotactic injections using a laser scan of the skull. arXiv 1410: 5914.

4. Grange P, Mitra PP (2011) Algorithmic choice of coordinates for injections into the brain: Encoding a neuroanatomical atlas on a grid. arXiv 1104: 2616.

5. Assi H, Candolfi M, Lowenstein PR, Castro MG (2013) Animal models of brain tumors. Intracranial Stereotactic Allografts and Xenografts. Humana Press Totowa, NJ, USA.

6. Paxinos G, Watson C (1977) The rat brain in stereotaxic coordinates. Academic Press, San Diego, USA.

7. Brunelli $R$ (2009) Template matching techniques in computer vision: Theory and practice. Wiley, Hoboken, USA 